\title{
Histone demethylases and their roles in cancer epigenetics.
}

\author{
Alexandra D'Oto', Qing-wu Tian², Andrew M Davidoff' ${ }^{1}$, Jun Yang ${ }^{1 *}$ \\ ${ }^{1}$ Department of Surgery, St Jude Children's Research Hospital, Memphis, USA. \\ ${ }^{2}$ Department of Clinical Laboratory, The Affiliated Hospital of Qingdao University, China.
}

\begin{abstract}
The genetic abnormalities that drive tumorigenesis are usually coupled with epigenetic alterations, such as DNA methylation and aberrant histone modifications, which may help oncogenic drivers accelerate cancer progression, metastasis, and therapy resistance. The discovery of histone demethylases has provided us new insight for understanding the epigenetic landscape of the chromatin environment of cancer cells. This review aims to summarize the current knowledge on the human histone lysine demethylases and their functions in cancers, and recent advances in development of small molecule inhibitors to target histone demethylases in cancer treatment.
\end{abstract}

Keywords: Epigenetics, Histone demethylase, Cancer.

\section{Introduction}

The genetic abnormalities that drive tumorigenesis are usually coupled with epigenetic alterations such as aberrant histone modifications including histone lysine methylations. Once thought to be an irreversible process, histone methylation has become an important focus in epigenetics and cancer research [1]. Histone methylation has been shown to be a key player in the regulation of gene expression and genetic stability, and not surprisingly, dysregulation of this highly conserved process occurs in various cancers [2]. There are two classes of enzymes involved in histone methylation: methyltransferases and demethylases. While methyltransferases are responsible for establishing methylation patterns, demethylases are capable of removing methyl groups not only from histones but other proteins as well $[3,4]$. The recent discovery of histone demethylases and their role in the regulation of posttranslational modifications of chromatin have greatly contributed to our understanding of epigenetics in tumorigenesis and have potentially provided additional therapeutic targets in the treatment of cancer [5]. Here we have summarized the recent advances in understanding the functions of histone demethylases in cancer and the potential therapeutic value to target histone demethylases in cancer.

\section{Classes of Histone Demethylases}

Histone methylation occurs on arginine and lysine residues found within histone tails. Arginine residues are mono- or dimethylated most extensively at their guanidinium groups, and methylation at these sites on $\mathrm{H} 3$ tails is associated with active transcription [6,7]. Arginine residue demethylation occurs via peptidyl arginine deiminase 4 (PADI4) which opposes methylation by converting arginine to citrulline [7].
This modification, however, is not considered demethylation, as conversion from arginine to citrulline results in loss of a methyl group without leaving a free arginine. Lysine residues can be either mono-, di- or trimethylated at the $\varepsilon$-nitrogen with methylation associated with either transcriptional activation or repression depending on the lysine location and level of methylation [4]. Di- and trimethylation at $\mathrm{H} 3 \mathrm{~K} 4$ is associated with enhanced gene expression whereas methylation at $\mathrm{H} 3 \mathrm{~K} 9, \mathrm{H} 3 \mathrm{~K} 27$ and H4K20 is associated with repression [8]. These methyl groups are able to be removed by true histone demethylases that are categorized into two families: amino oxidase homolog lysine demethylase 1 (KDM1) and JmjC domain-containing histone demethylases [1] (Table 1).

\section{KDM1 Family}

KDM1 family is composed of two members, KDM1A and KDM1B. KDM1A, also referred to as lysine-specific demethylase 1 (LSD1), was first described by Shi et al in 2004 [9]. This protein was found to be a highly conserved flavincontaining amino oxidase homolog that specifically removes the mono- and di-methylated lysine at lysine 4 or lysine 9 of H3, depending on the cellular context $[9,10]$. KDM1A contains a SWIRM domain, which has been identified in chromatin-modifying proteins [11]. KDM1A removes methyl groups via oxidation resulting in the formation of formaldehyde [9]. Several studies have shown that KDM1A demethylates specifically at $\mathrm{H} 3 \mathrm{~K} 4 \mathrm{me} 1$ and $\mathrm{H} 3 \mathrm{~K} 4 \mathrm{me} 2$ through the interaction between the tower domain of KDM1 A and CoREST, leading to transcriptional inactivation $[12,13]$. However, when complexed with androgen receptor, KDM1A demethylates $\mathrm{H} 3 \mathrm{~K} 9 \mathrm{me} 1$ and $\mathrm{H} 3 \mathrm{~K} 9 \mathrm{me} 2$, resulting in transcriptional activation [10]. KDM1A, however, is unable to demethylate trimethylated lysines; the oxidation reaction 
Table 1. Histone demethylases and the association with cancer

\begin{tabular}{|c|c|c|}
\hline HDM subfamily & Histone substrates & Association with cancer \\
\hline \multirow[b]{2}{*}{ KDM1A, LSD1, AOF2, BHC110 } & H3K4me1, H3K4me2 & \multirow{2}{*}{$\begin{array}{l}\text { Demethylates p53; Inhibits differentiation of } \\
\text { neuroblastoma and leukemia; Interacts with EWS/FLI1 } \\
\text { in osteosarcoma }\end{array}$} \\
\hline & H3K9me1, H3K9me2 & \\
\hline KDM1B, LSD2, AOF1 & H3K4me1, H3K4me2 & - \\
\hline KDM2A, FBXL11, JHDM1A & H3K36me1, H3K36me2 & $\begin{array}{l}\text { Mutation in intracranial germ cell tumor; Promotes lung } \\
\text { tumorigenesis }\end{array}$ \\
\hline \multirow{2}{*}{ KDM2B, FBXL10, JHDM1B } & H3K36me1, H3K36me2 & \multirow{2}{*}{$\begin{array}{l}\text { Leukemia transformation; Promotes tumor angiogenesis } \\
\text { Represses Ink } 4 \mathrm{~b} \text { tumor suppressor }\end{array}$} \\
\hline & H3K4me3* & \\
\hline KDM3A, JMJD1A, JHDM2A, TSGA & H3K9me1, H3K9me2 & $\begin{array}{l}\text { HIF1 targets; Regulates breast cancer invasion; Maintains } \\
\text { myeloma cell survival }\end{array}$ \\
\hline KDM3B, JMJD1B & H3K9me1, H3K9me2 & Deletion in MDS and AML \\
\hline JMJD1C & H3K9me1, H3K9me2* & Mutated in intracranial germ cell tumor \\
\hline \multirow{3}{*}{ KDM4A, JMJD2A, JHDM3A } & H3K9me2, H3K9me3 & \multirow{3}{*}{$\begin{array}{l}\text { Inhibits tumor suppressor CHD4; Induces site specific } \\
\text { copy gain; Regulates ERa in breast cancer }\end{array}$} \\
\hline & H3K36me2, H3K36me3 & \\
\hline & $\mathrm{H} 1.4 \mathrm{~K} 26 \mathrm{me} 2, \mathrm{H} 1.4 \mathrm{~K} 26 \mathrm{me} 3$ & \\
\hline \multirow{3}{*}{ KDM4B, JMJD2B, JHDM3B } & H3K9me2, H3K9me3 & \multirow{3}{*}{$\begin{array}{l}\text { HIF1 targets; Regulates MYC function in neuroblastoma, } \\
\text { ERa in breast cancer, AR in prostate cancer; } \\
\text { Leukemogenesis }\end{array}$} \\
\hline & H3K36me2, H3K36me3 & \\
\hline & H1.4K26me2, H1.4K26me3 & \\
\hline \multirow{3}{*}{ KDM4C, JMJD2C, JHDM3C, GASC1 } & H3K9me2, H3K9me3 & \multirow{3}{*}{$\begin{array}{l}\text { HIF1 targets; Amplified in lymphoma with JAK1; } \\
\text { Leukemogenesis }\end{array}$} \\
\hline & H3K36me2, H3K36me3 & \\
\hline & H1.4K26me2, H1.4K26me3 & \\
\hline KDM4D, JMJD2D, JHDM3D & H3K9me3 & - \\
\hline KDM5A, JARD1A, RBP2 & H3K4me2, H3K4me3 & $\begin{array}{l}\text { Interacta with } \mathrm{Rb} \text {; Inhibits tumor growth when deleted; } \\
\text { Drug resistance; Translocated to form oncofusion protein } \\
\text { with NUP98 in AML; AKT target }\end{array}$ \\
\hline KDM5B, JARD1B, PLU1 & $\mathrm{H} 3 \mathrm{~K} 4 \mathrm{me} 2, \mathrm{H} 3 \mathrm{~K} 4 \mathrm{me} 3$ & $\begin{array}{l}\text { Regulates AR in prostate cancer; Inhibits TGFb in breast } \\
\text { cancer }\end{array}$ \\
\hline KDM5C, JARD1C, SMCX & H3K4me2, H3K4me3 & $\begin{array}{l}\text { Mutated in renal clear cell carcinoma; Suppresses } \\
\text { heterochomatic ncRNA }\end{array}$ \\
\hline KDM5D, JARD1D, SMCY & H3K4me2, H3K4me3 & $\begin{array}{l}\text { Deleted in prostate cancer; KDM5D loss leads to } \\
\text { Docetaxal resistance in prostate cancer }\end{array}$ \\
\hline KDM6A, UTX & K3K27me2, H3K27me3 & Mutated in multiple tumor types \\
\hline KDM6B, JMJD3 & H3K27me2, H3K27me3 & Regulates NOTCH1 oncogene in ALL \\
\hline \multirow{2}{*}{ KDM7A, KIAA1718, JHDM1D } & H3K9me1, H3K9me2 & \multirow{2}{*}{ Inhibit tumor angiogenesis } \\
\hline & H3K27me1, H3K27me2 & \\
\hline KDM8, JMJD5 & H3K36me2* & - \\
\hline PHF8 & H3K9me1, H3K9me2 & $\begin{array}{l}\text { Promotes breast oncogenesis; Governs retinoic acid } \\
\text { response in AML }\end{array}$ \\
\hline PHF2, JHDM1E & H3K9me2* & Inhibit tumor initiation; Protein kinase A pathway \\
\hline \multirow{2}{*}{ NO66 } & H3K4me2, H3K4me3 & \multirow[t]{2}{*}{ 要 } \\
\hline & H3K36me2, H3K36me3 & \\
\hline
\end{tabular}

* Not consistent

in its catalytic domain depends on FAD and a lone pair of electrons from a lysine $\varepsilon$-nitrogen [5]. Non-histone proteins, such as p53, are also substrates for KDM1A.

Demethylation of K370me2 by KDM1A prevents the interaction of p53 with p53 binding protein 1 (53BP1), which represses the functions of $\mathrm{p} 53$, including induction of apoptosis [14]. Nicholson et al also demonstrated that KDM1A demethylates DNA cytosine-5-methyltransferase 1 (DNMT1), which leads to stabilization of DNMT1 so that DNMT1 may maintain DNA methylation patterns in embryonic stem cells [3]. Shortly after the discovery of KDM1A, another amine oxidase homolog was reported: KDM1B [15]. Like KDM1A, KDM1B is another FADdependent amino oxidase homolog containing a SWIRM domain and specifically targets $\mathrm{H} 3 \mathrm{~K} 4 \mathrm{me} 1$ and $\mathrm{H} 3 \mathrm{~K} 4 \mathrm{me} 2$ $[15,16]$. KDM1B, however, is unable to form a complex with CoREST as it lacks a tower domain [15]. The regulatory functions of KDM1B are still being studied, although more recent studies have shown KDM1B plays a role in maternal imprinting in oocytes and may have a part in activating NF$\kappa \mathrm{B}[16,17]$.

\section{The JMJC Domain-Containing Demethylases}

The second and largest group of the histone demethylases is the Jumonji C (JmJC) domain-containing demethylases. Of the identified JmJC domain proteins, nearly 20 are considered lysine specific demethylases [5]. These enzymes belong to the 2-oxoglutarate-dependent dioxygenases, and require $\mathrm{Fe}^{2+}$ 
and oxygen in order to undergo the hydroxylation necessary to remove methyl groups. Unlike KDM1, this family of enzymes is capable of removal of trimethylations [18]. There are several different subfamilies of the JmjC KDMs that have been identified including KDM2, KDM3, KDM4, KDM5, KDM6 and others.

The KDM2/FBXL subfamily consists of 2 members: KDM2A and KDM2B. While both of these enzymes function to specifically demethylate $\mathrm{H} 3 \mathrm{~K} 36 \mathrm{me} 2[19,20]$, KDM2B is also able to demethylate H3K4me3 [21]. KDM2B regulates $\mathrm{p} 15^{\text {Ink4b }}$ through demethylation at $\mathrm{H} 3 \mathrm{~K} 36 \mathrm{me} 2$, leading to repression at that locus(19). Knockdown of KDM2B has been shown to lead to induction of cellular senescence in a p53- and RB- dependent manner [19]. One study proposed KDM2B may protect against oxidative stress by inhibiting ROS-mediated signaling while another study indicated KDM2A may suppress the NF- $\mathrm{KB}$ pathway $[22,23]$.

KDM3A and its two human homologs KDM3B and JMJD1C make up the KDM3/JMJD1C subfamily. While JMJD1C has no histone demethylase activity, KDM3A and KDM3B are specific for removing $\mathrm{H} 3 \mathrm{~K} 9 \mathrm{me} 1$ and $\mathrm{H} 3 \mathrm{~K} 9 \mathrm{me} 2$. While KDM3A is shown to act on androgen receptors in a liganddependent manner and to have a role in spermatogenesis and metabolism [24], KDM3B seems also involved in spermatogenesis [25].

Specific demethylation of H3K9me2, H3K9me3, H3K36me2 and H3K36me3 is catalyzed by the KDM4 subfamily. Although much remains unknown, there are several studies that reveal this subfamily's involvement in activating gene expression. In particular, $\mathrm{KDM} 4 \mathrm{C}$ has been shown to increase the amount of euchromatin available for transcription [26]. NOTCH1 signaling also appears to be directly influenced by KDM4C [27]. More recently, the direct interaction between $\mathrm{N}-\mathrm{Myc}$ and KDM4B has been established [28]. Interestingly, KDM4A was found to be associated with translation machinery and regulates protein synthesis [29].

KDM5 subfamily has catalytic activity at $\mathrm{H} 3 \mathrm{~K} 4 \mathrm{me} 2$ and H3K4me3. KDM5A has been implicated in Notch/RBP-J complex gene silencing through direct interaction with RBP-J [30]. Other studies have pointed to its role in gene repression via PRC2 complex [31]. KDM5B expression, however, seems to be concentrated in the testes and associates with the androgen receptor to regulate transcription [32].

KDM6A/UTX and KDM6B/JMJD3 act on H3K27me3, a mark of gene repression [33]. KDM6A acts as a regulator of the cell cycle in a RB-dependent manner and counteracts growth signals by maintaining RB-binding proteins to induce cell cycle arrest [34]. The role of KDM6B is more complicated as current research posits contradictory functions of this enzyme. KDM6B is not only shown to associate with p53, but is seen recruited to promoter and enhancer elements that correspond to 263 of p53 target genes [35]. KDM6B has also been shown to stabilize nuclear $\mathrm{p} 53$ through direct interaction as well as increase the expression of $\mathrm{p} 16^{\mathrm{INK} 4 \mathrm{~A}}$ and $\mathrm{p} 14^{\mathrm{ARF}}$, found at the INK4A-ARF locus [36,37]. Other studies have shown KDM6B to control the expression of the NOTCH1 oncogene in leukemia [38].

\section{Role of Histone Demethylases in Cancer}

Given their various functions in transcriptional activity, it is no surprise that alteration and aberrant expression of histone demethylases have been noted in various cancers. KDM1A overexpression has also been found in poorly differentiated neuroblastoma [39]. KDM1A was found to prevent the differentiation process and maintain the malignant phenotype in neuroblastoma, perhaps through gene silencing via the REST/CoREST complex. KDM1A is a key effector of the differentiation block in MLL-AF9 leukemia [40]. Inhibition of the KDM1A demethylase reactivates the all-trans-retinoic acid differentiation pathway in acute myeloid leukemia [41]. One study shows that KDM1A is a subunit of the NuRD complex and targets the metastasis programs in breast cancer [42]. In addition, LSD1 directly interacts with oncofusion protein EWS/FWI1, maintaining EWS/FWI1 activating and repressive activity in Ewing's sarcoma [43]. The direct interaction of LSD1 with p53 decreases the activity of p53, including decreased expression of $\mathrm{p} 21$ and is associated with tumorigenesis [14]. On the other hand, KDM1B has not been found to be altered in many cancers, although studies suggest KDM1B may function as an E3 ubiquitin ligase, leading to the proteasomal degradation of OGT, an enzyme often increased in several diseases, including cancer and diabetes [44].

Ectopic expression of either KDM2A or KDM2B led to immortalization of mouse embryonic fibroblasts through the promotion of RB phosphorylation [45]. KDM2A promotes lung tumorigenesis by epigenetically enhancing ERK $1 / 2$ signaling [46]. KDM2B is upregulated in leukemic stem cells and is required in their neoplastic transformation [47]. Increased KDM2B in pancreatic cancers correlates to poorer prognosis and increased tumor aggression [48]. Furthermore, KDM2B promotes angiogenesis in bladder cancer via induction of EZH2 of the PRC2 complex [20]. KDM2B represses Ink4b in mouse fibroblasts and alteration in KDM2B expression results in Ras-induced transformation [19].

Very few studies have focused on the KDM3 subfamily. KDM3A is a target of hypoxia inducible factor 1 [49]. KDM3A has been shown to enhance tumor growth [50], regulate breast cancer cell invasion and apoptosis [4], and maintain myeloma cell survival [51]. A frequently deleted region in MDS and AML is 5q31, which corresponds to the location of nuclear protein 5qNCA (LOC51780) [52]. $5 q$ qNCA contains a portion of the KDM3B gene, thus KDM3B is thought to play a role in tumor suppression [52]. However, one study shows that KDM3B is involved in transcriptional activation of the LMO2 oncogene in leukemia [53].

Substantial evidence points towards the KDM4 subfamily as proto-oncogenic. KDM4A and its relatives KDM4B and KDM4C have been shown to stimulate androgen receptordependent growth, and their overexpression is seen in prostate cancer [54]. ER $\alpha$-positive breast cancer cells have been shown to have upregulated levels of KDM4A and KDM4B, while triple-negative breast cancer cells have upregulated KDM4C [54]. KDM4A and KDM4B interact with $\mathrm{ER} \alpha$ to promote transcription of ER $\alpha$-target genes such as MYC [55]. KDM4B 
is a direct target of ER $\alpha$ and epigenetically regulates cell cycle progression of breast cancer cells [56]. KDM4A promotes cellular transformation by blocking cellular senescence through transcriptional repression of the tumor suppressor CHD5 [57]. Interestingly, a recent study shows that KDM4A induces site-specific copy gain and re-replication of regions amplified in tumors [58] and further, this group reports that hypoxia is able to drive transient site-specific copy gain and drug-resistant gene expression via KDM4A [59]. KDM4B has recently been found to play an important role in the $\mathrm{N}-\mathrm{Myc}$ pathway of neuroblastoma cells [28]. Compared to more differentiated tumors such as ganglioneuroblastoma and ganglioneuroma, neuroblastoma expresses higher levels of KDM4B [28]. Abnormalities of KDM4C have been noted in several cancer types, including $\mathrm{B}$ cell and Hodgkin lymphoma. Amplification of 9p24 region, which contains the genes for $\mathrm{KDM} 4 \mathrm{C}$ and JAK2, is noted in these cancer cell lines [60]. This amplicon is also seen in esophageal cancer and metastatic lung sarcomatoid carcinoma [27]. Through the demethylation of $\mathrm{H} 3 \mathrm{~K} 9 \mathrm{me} 3, \mathrm{KDM} 4 \mathrm{C}$ is shown to be a co-activator of HIF- $1 \alpha$ and is required for the proliferation and metastasis in breast cancer [61]. The KDM4 subfamily members KDM4A, KDM4B and KDM4C were also newly found to be required for the transformation of leukemia cells by enhancing the expression of IL-3 receptor- $\alpha$, a key initiator in the JAK-STAT pathway, in MLL-AF9 translocated AML, a highly aggressive leukemia with poor prognosis [62]. This study additionally purports that KDM4A, KDM4B and KDM4C may have interdependent, coordinated functions within the cells [62]. Little is reported on KDM4D and the available studies are contradictory. KDM4D appears to be a coactivator of the androgen receptor and required for colon cancer progression; however, KDM4D also has been shown to activate p53-dependent transcription [54]. KDM4D's role in tumorigenesis thus remains to be elucidated.

KDM5A is overexpressed in multiple cancers. Loss of KDM5A suppresses tumorigenesis in mice lacking $\mathrm{Rb} 1$ or Men1 [63]. KDM5A is considered to be required for reversible drug resistance in cancer cell subpopulations [64]. The development of drug-tolerance is thought to arise from the upregulation of IGF-1, a growth factor often associated with cancer cell proliferation and metastasis [65]. The carboxy-terminal PHD finger of KDM5A fused to a common fusion partner nucleoporin-98 (NUP98) as identified in human leukemias, generated potent oncoproteins that arrested haematopoietic differentiation and induced acute myeloid leukemia in murine models [65]. However, a recent study shows that KDM5A is an AKT target and that phosphorylation of KDM5A regulates its nuclear localization and promoter occupancy, indicating KDM5A may play a tumor suppressive function in this context [66].

Particularly, KDM5B is overexpressed in prostate cancer and the role of $\mathrm{KDM} 5 \mathrm{~B}$ in prostate cancer is thought to be associated to its coactivation of the androgen receptor [32]. Increased expression of KDM5B is also seen in breast cancer and testis cancer [67]. KDM5B is often upregulated in luminal breast cancer cells and is thought to repress the cell cycle inhibitor TGF $\beta$ [68]. Additionally, heterozygous missense mutation at $\mathrm{K} 1435 \mathrm{R}$ in KDM5B is observed in HCC2157 breast cell line and may code for a gain-of-function enzyme with increased demethylase activity [68]. KDM5C inactivation led to the unrestrained expression of heterochromatic noncoding RNAs (ncRNAs) that in turn triggered genomic instability in clear cell renal cell carcinoma [69].

The first reported histone demethylase found to be mutated in cancers was KDM6A [70]. Investigation of somatic inactivating mutations in KDM6A revealed somatic mutational prevalence in several types of malignancies including adult and pediatric cancers [71,72]. KDM6A target genes include pRB-binding proteins, thus the disruption of KDM6A leads to dysregulation of the cell cycle [34]. KDM6B appears to have a more context-dependent role in cancers. One study showed KDM6B plays a role in the activation of transcription of anti-apoptotic protein $\mathrm{Bcl} 2$ in an ER $\alpha$-dependent manner in breast cancer cells [73]. The apparent role of KDM6B in the initiation and maintenance of T-ALL also established [38]. On the other hand, increased expression of KDM6B increased the expression of $\mathrm{p} 16^{\mathrm{INK} 4 \mathrm{~A}}$ and $\mathrm{p} 14^{\mathrm{ARF}}$ at the $I N K 4 A-$ $A R F$ locus in fibroblasts [36]. KDM6B expression was also found to stabilize nuclear p53 and induced expression of 21 independently of the p16, p14 or p53 pathway to aid in partial cell cycle arrest and differentiation in germline stem cells, preventing tumorigenesis [37].

\section{KDM Inhibitors}

Several types of KDM1A inhibitors have been proposed. Substrate analogs and polyamine analogs were developed to target the catalytic domains of KDM1A. Oligoamine analogs were found to induce re-expression of aberrantly silenced genes in HCT116 cells [2]. In addition, (bis) urea and (bis) thiourea inhibitors showed similar results in Calu-6 lung carcinoma cells [74]. Other inhibitors tested include antiMAO agents due to the striking similarity between KDM1A and MAO sequences [2]. These agents selectively modify the benzoyl and benxylamino groups found in the active site of KDM1A [75]. Although several KDM1A inhibitors have been synthesized, only a few show clinical utility. A highly specific, non-competitive KDM1A inhibitor, HCI-2509 (SP2509), has been effective in inducing apoptosis in Ewing sarcoma cell lines, particularly those that express the EWSFLI1 fusion protein, alone and in combination with other anticancer agents [76]. HCI-2509 produces similar effects on other cancer cell lines, such as poorly differentiated endometrial carcinoma and AML [77,78]. In fact, KDM1A inhibitor GSK2879552 is currently in a Phase I drug trial to determine dosing for patients with relapsed/refractory AML (https://clinicaltrials.gov/ct2/show/NCT02177812).

Few inhibitors have been identified to target the JmjC domain-containing histone demethylases. N-oxalylglycine and its derivatives inhibit KDM2A, KDM2C and KDM2D and increase histone lysine methylation at KDM2 target sites; however these drugs lack specificity as it acts on other $\alpha$-ketoglutarate dependent enzymes [79]. Selective inhibitors for KDM5 and KDM6 subfamilies have been discovered (CPI-455 and GSK-J1/GSK-J4, respectively) [80,81], but the off-target effects of these drugs are yet to be elucidated. As of now, no potent selective inhibitor of either KDM3 or KDM4 has been found. 
Despite the limited number of studies, histone demethylase inhibitors appear to be promising agents for anticancer therapy. These enzymes, however, have shown to have complex biological interactions within the cell, so determining the downstream effects of histone demethylase inhibition in vivo will need to be established.

\section{Conclusion}

With advances of novel therapeutics, cancer, at least some types of malignancies, will be slowly progressing from a fatal disease to a chronically manageable and treatable disease. Our focus has shifted from symptom management to eradication. As we strive to make this goal a reality, researchers must delve deeper into the mechanisms of carcinogenesis. Epigenetics has shed new light on the road to curing cancer and has opened the door to newer treatments that can focus on rewriting the cancer epigenome. With the discovery of histone demethylases in 2004, we now know that histone methylation is not an irreversible process, but rather another testament to the dynamicity of the human genome. As the link between histone demethylases and tumorigenesis is further reinforced, the potential for these enzymes as a therapeutic target continues to strengthen. Histone demethylases not only target methylated sites on histone tails but also interact with methylated sites on non-histone proteins, such as p53. As we persist in our search for potent yet selective cancer therapies, histone demethylases are a reservoir of untapped potential. Future studies may reveal more on the mechanisms of neoplastic transformation and provide invaluable insight as to how we can stop it.

\section{Acknowledgement}

This work was supported by the Assisi Foundation of Memphis, the US Public Health Service Childhood Solid Tumor Program Project Grant No. CA23099, the Cancer Center Support Grant No. 21766 from the National Cancer Institute, and by the American Lebanese Syrian Associated Charities (ALSAC) (to ADM).

\section{Author's Contribution}

$\mathrm{ADO}, \mathrm{QT}, \mathrm{ADM}$ and JY were involved in manuscript writing.

\section{References}

1. Shi Y, Whetstine JR. Dynamic regulation of histone lysine methylation by demethylases. Mol Cell 2007; 25: 1-14.

2. Rotili D, Mai A. Targeting histone demethylases: A new avenue for the fight against cancer. Genes Cancer 2011; 2: 663-679.

3. Nicholson TB, Chen T. LSD1 demethylates histone and nonhistone proteins. Epigenetics 2009; 4: 129-132.

4. Ramadoss S, Guo G, Wang CY. Lysine demethylase KDM3A regulates breast cancer cell invasion and apoptosis by targeting histone and the non-histone protein $\mathrm{p} 53$. Oncogene. 2016.

5. Hojfeldt JW, Agger K, Helin K. Histone lysine demethylases as targets for anticancer therapy. Nat Rev Drug Discov 2013; 12: $917-930$.

6. Bedford MT, Clarke SG. Protein arginine methylation in mammals: Who, what and why. Mol Cell 2009; 33: 1-13.
7. Cuthbert GL. Histone deimination antagonizes arginine methylation. Cell 2004; 118: 545-553.

8. Margueron R, Trojer P, Reinberg D. The key to development: Interpreting the histone code? Curr Opin Genet Dev 2005; 15: $163-176$

9. Shi Y. Histone demethylation mediated by the nuclear amine oxidase homolog LSD1. Cell 2014; 119: 941-953.

10. Wissmann M. Cooperative demethylation by JMJD2C and LSD1 promotes androgen receptor-dependent gene expression. Nat Cell Biol 2007; 9: 347-353.

11. Aravind L, Iyer LM. The SWIRM domain: A conserved module found in chromosomal proteins points to novel chromatin-modifying activities. Genome Biol 2002; 3: 39.

12. Lee MG, Wynder C, Cooch N, et al. An essential role for CoREST in nucleosomal histone 3 lysine 4 demethylation. Nature 2005; 437: 432-435.

13. Yang M. Structural basis for CoREST-dependent demethylation of nucleosomes by the human LSD1 histone demethylase. Mol Cell 2006; 23: 377-387.

14. Huang J. p53 is regulated by the lysine demethylase LSD1. Nature 2007; 449: 105-108.

15. Karytinos A. A novel mammalian flavin-dependent histone demethylase. J Biol Chem 2009; 284: 17775-17782.

16. Ciccone DN. KDM1B is a histone H3K4 demethylase required to establish maternal genomic imprints. Nature 2009; 461: 415-418.

17. van Essen D, Zhu Y, Saccani S. A feed-forward circuit controlling inducible NF-kappaB target gene activation by promoter histone demethylation. Mol Cell 2010; 39: 750760 .

18. Cloos PA, Christensen J, Agger K, et al. Erasing the methyl mark: histone demethylases at the center of cellular differentiation and disease. Genes Dev 2008; 22: 1115-1140.

19. He J, Kallin EM, Tsukada Y, et al. The H3K36 demethylase $\mathrm{Jhdm} 1 \mathrm{~b} / \mathrm{Kdm} 2 \mathrm{~b}$ regulates cell proliferation and senescence through p15(Ink4b). Nat Struct Mol Biol 2008; 15: 11691175 .

20. Kottakis F. FGF-2 regulates cell proliferation, migration and angiogenesis through an NDY1/KDM2B-miR-101-EZH2 pathway. Mol Cell 2011; 43: 285-298.

21. Frescas D, Guardavaccaro D, Bassermann F, et al. JHDM1B/ FBXL10 is a nucleolar protein that represses transcription of ribosomal RNA genes. Nature 2007; 450: 309-313.

22. Lu T. Regulation of NF-kappaB by NSD1/FBXL11dependent reversible lysine methylation of $\mathrm{p} 65$. Proc Natl Acad Sci 2010; 107: 46-51.

23. Polytarchou C, Pfau R, Hatziapostolou M, et al. The JmjC domain histone demethylase Ndyl regulates redox homeostasis and protects cells from oxidative stress. Mol Cell Biol 2008; 28: 7451-7464.

24. Okada Y, Scott G, Ray MK, et al. Histone demethylase JHDM2A is critical for Tnp1 and Prm1 transcription and spermatogenesis. Nature 2007; 450: 119-123. 
25. Liu Z. Knockout of the histone demethylase Kdm3b decreases spermatogenesis and impairs male sexual behaviors. Int $\mathrm{J}$ Biol Sci 2015; 11: 1447-1457.

26. Cloos PA. The putative oncogene GASC1 demethylates triand dimethylated lysine 9 on histone H3. Nature 2016; 442: 307-311.

27. Liu G. Genomic amplification and oncogenic properties of the GASC1 histone demethylase gene in breast cancer. Oncogene 2009; 28: 4491-4500.

28. Yang J. The role of histone demethylase KDM4B in Myc signaling in neuroblastoma. J Natl Cancer Inst 2015; 107: 80 .

29. Van Rechem C. Lysine demethylase KDM4A associates with translation machinery and regulates protein synthesis. Cancer Discov 2015; 5: 255-263.

30. Liefke R. Histone demethylase KDM5A is an integral part of the core Notch-RBP-J repressor complex. Genes Dev 2010; 24: 590-601.

31. Pasini D. Coordinated regulation of transcriptional repression by the RBP2 H3K4 demethylase and Polycomb-Repressive Complex 2. Genes Dev 2008; 22: 1345-1355.

32. Xiang Y. JARID1B is a histone H3 lysine 4 demethylase upregulated in prostate cancer. Proc Natl Acad Sci USA 2007; 104: 19226-19231.

33. Margueron R, Reinberg D. The Polycomb complex PRC2 and its mark in life. Nature 2011; 469: 343-349.

34. Wang JK. The histone demethylase UTX enables RBdependent cell fate control. Genes Dev 2010; 24: 327-332.

35. Williams K. The histone lysine demethylase JMJD3/KDM6B is recruited to $\mathrm{p} 53$ bound promoters and enhancer elements in a p53 dependent manner. PLoS One 2014; 9: e96545.

36. Agger K. The H3K27me3 demethylase JMJD3 contributes to the activation of the INK4A-ARF locus in response to oncogene- and stress-induced senescence. Genes Dev 2009; 23: $1171-1176$

37. Ene CI. Histone demethylase Jumonji D3 (JMJD3) as a tumor suppressor by regulating p53 protein nuclear stabilization. PLoS One 2012; 7: e51407.

38. Ntziachristos P. Contrasting roles of histone 3 lysine 27 demethylases in acute lymphoblastic leukaemia. Nature 2014; 514: 513-517.

39. Schulte JH. Lysine-specific demethylase 1 is strongly expressed in poorly differentiated neuroblastoma: implications for therapy. Cancer Res 2009; 69: 2065-2071.

40. Harris WJ. The histone demethylase KDM1A sustains the oncogenic potential of MLL-AF9 leukemia stem cells. Cancer Cell 2012; 21: 473-487.

41. Schenk T. Inhibition of the LSD1 (KDM1A) demethylase reactivates the all-trans-retinoic acid differentiation pathway in acute myeloid leukemia. Nat Med 2012; 18: 605-611.

42. Wang Y. LSD1 is a subunit of the NuRD complex and targets the metastasis programs in breast cancer. Cell 2009; 138: 660-672.
43. Sankar S. Reversible LSD1 inhibition interferes with global EWS/ETS transcriptional activity and impedes Ewing sarcoma tumor growth. Clin Cancer Res 2014; 20: 45844597.

44. Yang Y, Yin X, Yang H, et al. Histone demethylase LSD2 acts as an E3 ubiquitin ligase and inhibits cancer cell growth through promoting proteasomal degradation of OGT. Mol Cell 2015; 58: 47-59.

45. Pfau R. Members of a family of JmjC domain-containing oncoproteins immortalize embryonic fibroblasts via a JmjC domain-dependent process. Proc Natl Acad Sci USA 2008; 105: 1907-1912.

46. Wagner KW. KDM2A promotes lung tumorigenesis by epigenetically enhancing ERK1/2 signaling. J Clin Invest 2013; 123: 5231-5246.

47. He J, Nguyen AT, Zhang Y. KDM2b/JHDM1b, an H3K36me2-specific demethylase, is required for initiation and maintenance of acute myeloid leukemia. Blood 2011; 117: 3869-3880.

48. Tzatsos A. KDM2B promotes pancreatic cancer via Polycomb-dependent and -independent transcriptional programs. J Clin Invest 2013; 123: 727-739.

49. Beyer S, Kristensen MM, Jensen KS, et al. The histone demethylases JMJD1A and JMJD2B are transcriptional targets of hypoxia-inducible factor HIF. J Biol Chem 2008; 283: 36542-36552.

50. Krieg AJ. Regulation of the histone demethylase JMJD1A by hypoxia-inducible factor 1 alpha enhances hypoxic gene expression and tumor growth. Mol Cell Biol 2010; 30: 344353.

51. Ohguchi H. The KDM3A-KLF2-IRF4 axis maintains myeloma cell survival. Nat Commun 2016; 7: 10258.

52. Hu Z. A novel nuclear protein, 5qNCA (LOC51780) is a candidate for the myeloid leukemia tumor suppressor gene on chromosome 5 band q31. Oncogene 2001; 20: 6946-6954.

53. Kim JY. KDM3B is the H3K9 demethylase involved in transcriptional activation of $1 \mathrm{mo} 2$ in leukemia. Mol Cell Biol 2012; 32: 2917-2933.

54. Berry WL, Janknecht R. KDM4/JMJD2 histone demethylases: Epigenetic regulators in cancer cells. Cancer Res 2013; 73: 2936-2942.

55. Kawazu M. Histone demethylase JMJD2B functions as a cofactor of estrogen receptor in breast cancer proliferation and mammary gland development. PLoS One 2011; 6: e17830.

56. Yang J. The histone demethylase JMJD2B is regulated by estrogen receptor alpha and hypoxia, and is a key mediator of estrogen induced growth. Cancer Res 2010; 70: 6456-6466.

57. Mallette FA, Richard S. JMJD2A promotes cellular transformation by blocking cellular senescence through transcriptional repression of the tumor suppressor CHD5. Cell Rep 2012; 2: 1233-1243.

58. Black JC. KDM4A lysine demethylase induces site-specific copy gain and re-replication of regions amplified in tumors. Cell 2013; 154: 541-555. 
59. Black JC. Hypoxia drives transient site-specific copy gain and drug-resistant gene expression. Genes Dev 2015; 29: 1018-1031.

60. Rui L. Cooperative epigenetic modulation by cancer amplicon genes. Cancer Cell 2010; 18: 590-605.

61. Luo W, Chang R, Zhong J, et al. Histone demethylase JMJD2C is a coactivator for hypoxia-inducible factor 1 that is required for breast cancer progression. Proc Natl Acad Sci USA 2012; 109: E3367-3376.

62. Agger K. Jmjd2/Kdm4 demethylases are required for expression of Il3ra and survival of acute myeloid leukemia cells. Genes Dev 2016; 30: 1278-1288.

63. Lin W. Loss of the retinoblastoma binding protein 2 (RBP2) histone demethylase suppresses tumorigenesis in mice lacking Rb1 or Men1. Proc Natl Acad Sci USA 2011; 108: 13379-13386.

64. Sharma SV. A chromatin-mediated reversible drug-tolerant state in cancer cell subpopulations. Cell 2010; 141: 69-80.

65. Wang GG. Haematopoietic malignancies caused by dysregulation of a chromatin-binding PHD finger. Nature 2009; 459: 847-851.

66. Spangle JM. PI3K/AKT Signaling Regulates H3K4 Methylation in Breast Cancer. Cell Rep 2016; 15: 2692-2704.

67. Barrett A. PLU-1 nuclear protein, which is upregulated in breast cancer, shows restricted expression in normal human adult tissues: a new cancer/testis antigen? Int J Cancer 2012; 101: 581-588.

68. Yamamoto S. JARID1B is a luminal lineage-driving oncogene in breast cancer. Cancer Cell 2014; 25: 762-777.

69. Rondinelli B. Histone demethylase JARID1C inactivation triggers genomic instability in sporadic renal cancer. J Clin Invest 2015; 125: 4625-4637.

70. van Haaften G. Somatic mutations of the histone H3K27 demethylase gene UTX in human cancer. Nat Genet 2009; 41: 521-523.
71. Huether R. The landscape of somatic mutations in epigenetic regulators across 1,000 paediatric cancer genomes. Nat Commun 2014; 5: 3630.

72. Kandoth C. Mutational landscape and significance across 12 major cancer types. Nature 2013; 502: 333-339.

73. Svotelis A. H3K27 demethylation by JMJD3 at a poised enhancer of anti-apoptotic gene BCL2 determines ER alpha ligand dependency. EMBO J 2011; 30: 3947-3961.

74. Sharma SK. (Bis) urea and (bis) thiourea inhibitors of lysinespecific demethylase 1 as epigenetic modulators. J Med Chem 2010; 53: 5197-5212.

75. Ueda R. Identification of cell-active lysine specific demethylase 1-selective inhibitors. J Am Chem Soc 2009; 131: 17536-17537.

76. Theisen ER, Pishas KI, Saund RS, et al. (2016) Therapeutic opportunities in Ewing sarcoma: EWS-FLI inhibition via LSD1 targeting. Oncotarget 2016; 7: 17616-17630.

77. Fiskus W. Highly effective combination of LSD1 (KDM1A) antagonist and pan-histone deacetylase inhibitor against human AML cells. Leukemia 2014; 28: 2155-2164.

78. Theisen ER. Reversible inhibition of lysine specific demethylase 1 is a novel anti-tumor strategy for poorly differentiated endometrial carcinoma. BMC Cancer 2014; 14: 752 .

79. Hamada S. Synthesis and activity of N-oxalylglycine and its derivatives as Jumonji C-domain-containing histone lysine demethylase inhibitors. Bioorg Med Chem Lett 2009; 19: 2852-2855.

80. Vinogradova M. An inhibitor of KDM5 demethylases reduces survival of drug-tolerant cancer cells. Nat Chem Biol 2016; 12: 531-538.

81. Kruidenier L. A selective jumonji H3K27 demethylase inhibitor modulates the proinflammatory macrophage response. Nature 2012; 488: 404-408.

\section{Correspondence to:}

Jun Yang,

Department of Surgery,

St Jude Children's Research Hospital,

Memphis,

USA.

Tel: 901-595-5008

E-mail: Jun.Yang2@stjude.org 